\title{
3D printing orthopedic scoliosis braces: a test comparing FDM with thermoforming
}

\author{
Davide Felice Redaelli ${ }^{1}$ (D) - Valentina Abbate ${ }^{2,3}$ (D) Fabio Alexander Storm ${ }^{4}$ (D) Alfredo Ronca $^{2}$ (D) \\ Andrea Sorrentino $^{2}$ (D) Cristina De Capitani $^{2}$ (D) Emilia Biffi $^{4}$ (D) Luigi Ambrosio $^{2}$ (D) Giorgio Colombo $^{1}$ (D) \\ Paolo Fraschini ${ }^{4}$ (D)
}

Received: 18 May 2020 / Accepted: 7 September 2020 / Published online: 15 October 2020

(C) The Author(s) 2020

\begin{abstract}
In recent years, 3D printing gained considerable attention in the orthopedic sector. This work evaluates the feasibility of producing orthopedic scoliosis braces by $3 \mathrm{D}$ printing, comparing performance and costs with classical thermoforming procedures. Critical parameters, such as manufacture time, mechanical properties, weight, and comfort are carefully considered. Polyethylene terephthalate glycol-modified (PETG) was selected among the several filaments materials present on the market. Printed samples were analyzed with electronic microscope, tensile, and impact tests and compared with thermoformed polyethylene (PE) and polypropylene (PP) samples. Moreover, a cost analysis was carried out for the specific application. The thermoformed brace of a volunteer patient affected by scoliosis was reproduced using reverse-engineering techniques. The model was then printed as a single piece and postprocessed by an expert orthotist. Subsequently, the patient wore the brace in a pilot case to compare comfort and mechanical effectiveness. Results show that the $3 \mathrm{D}$ printing fabrication method is able to provide a valid alternative to the current fabrication methods, being also very competitive in terms of costs. The morphological analysis does not show critical defects in 3D printed samples, while the mechanical tests highlighted their anisotropy, with an overall brittleness of PETG samples in the direction orthogonal to the fibers. However, in terms of mechanical stresses, a back brace should never reach the polymer yield stress, otherwise the shape would be modified and the therapeutic effect could be compromised. Finally, the patient reported the perception of improved support and no significant comfort differences compared with the thermoformed brace.
\end{abstract}

Keywords 3D printing $\cdot$ FDM $\cdot$ Manufacturing process $\cdot$ Mechanical characterization $\cdot$ PETG $\cdot$ Orthopedic scoliosis brace

Davide Felice Redaelli and Valentina Abbate contributed equally to this work.

Davide Felice Redaelli

davidefelice.redaelli@polimi.it

Giorgio Colombo

giorgio.colombo@polimi.it

Valentina Abbate

valentina.abbate@unina.it

Fabio Alexander Storm

fabio.storm@lanostrafamiglia.it

Alfredo Ronca

alfredo.ronca@cnr.it

Andrea Sorrentino

andrea.sorrentino@cnr.it

Cristina De Capitani

cristina.decapitani@cnr.it
Emilia Biffi

emilia.biffi@lanostrafamiglia.it

Luigi Ambrosio

luigi.ambrosio@cnr.it

Paolo Fraschini

paolo.fraschini@lanostrafamiglia.it

1 Department of Mechanical Engineering, Politecnico di Milano, Via G. La Masa, 1, 20156 Milano (MI), Italy

2 Institute of Polymers, Composites and Biomaterials, National Research Council of Italy, Via Previati 1/C, 23900 Lecco, Italy

3 Department of Chemical, Materials and Industrial Production Engineering (DICMaPI), University of Naples Federico II, Piazzale V. Tecchio 80, 80125 Napoli (NA), Italy

4 Scientific Institute IRCCS E. Medea, Via Don Luigi Monza, 20, 23842 Bosisio Parini (LC), Italy 


\section{Introduction}

Long term orthotic bracing is a very common nonsurgical therapy for the treatment of spine diseases [1,2]. Although there is evidence for the use of physiotherapy to address moderate spinal deformities, such as idiopathic scoliosis, brace treatment is preferred, often in combination with scoliosis specific daily rehabilitation, especially during the pubertal growth spurt [3, 4]. Scoliosis is defined as a structural abnormal curvature of the spine, typically over $10^{\circ}$ for Cobb measurement in the frontal plane. Depending on type, localization, structural rigidity, and severity of scoliosis, the effects of orthopedic braces can be limited to stabilization or extended also to correction of spine and chest deformities. Braces are shaped as to produce forces in definite anatomical regions keeping others unloaded, typically with a three-point bending system configuration $[5,6]$.

The design and manufacturing of a customized scoliosis brace is a complex process. Orthotists rely on personal experience and patients expectations to design an effective device with acceptable comfort. There is currently no unique standard for producing scoliosis braces. All manufacturing methods are currently based on producing a positive mold of the patient's body [4]. The mold can be realized either using plaster of Paris or via 3D scanning and computerized milling. In both cases, the orthotist manually adjusts the mold, sculpting and smoothing some regions according to medical prescriptions, manual measurements, and experience. The brace is then obtained by wrapping the positive mold with a preheated polymer sheet (thermoforming process). Sometimes, vacuum is used to improve adhesion. The brace is finalized by cropping and sanding the edges and applying straps for closing [7]. All these activities are currently performed manually by expert technicians with direct application on the patient. Thermoforming the plastic sheet does not allow to finely control the thickness and to obtain complex shapes or textures, with limitations of functionality, aesthetics, and comfort. Discomfort and unpleasantness lower the acceptance of braces by young patients, affecting their compliance to the therapy. In addition, due to the custom nature of these devices and the evolving conditions related to deformity correction and growth, each brace requires a new positive mold, whose disposal causes issues of waste management and costs.

Recently, the introduction of additive manufacturing, also known as three-dimensional printing (3DP), has shown a great potential for obtaining patient-specific solutions in the medical field $[8,9]$. This technique enables to create complex and customized devices based on digital models [10-12]. 3DP is environmentally friendly, produces limited waste material, and does not require molds. Each part can be customized and replicated without additional preparation cost. Less handwork is needed, reducing the risks of human errors and improving interoperator repeatability [13, 14]. Several 3DP methods have been applied in healthcare, such as poly jet modeling (PJM), selective laser sintering (SLS), stereolithography (SLA), and fused deposition modeling (FDM).

Currently, the major limiting factors for producing large orthotic devices by $3 \mathrm{DP}$ are processing times and high equipment costs (in particular, for PJM and SLS). However, among these technologies, FDM is the least expensive and the most suitable to produce scoliosis braces, despite a slightly lower dimensional accuracy. A few papers proposed the use of 3DP to fabricate orthotic devices $[15,16]$. Most of these studies focused on the possibility of obtaining devices with accurate shapes and geometrical dimensions, but only few considerations related to costs and mechanical performances of these devices are reported. Moreover, information on the mechanical properties in filament datasheets often refer to the bulk material before 3DP, while mechanical characterization of printed parts is scarce and mainly focused on tensile properties [17]. Furthermore, impact tests are important to quantify the toughness of a material, by measuring the amount of energy absorbed during high-strain-rate conditions before failure. This behavior certainly changes with different production technologies, such as 3DP [18]. For these reasons, it is not yet possible to predict how the combination of virtual modeling and additive manufacturing processes affects the mechanical properties of a $3 \mathrm{D}$ printed brace.

This work aims to evaluate the feasibility of producing a patient-specific orthopedic scoliosis brace by $3 \mathrm{DP}$ and to report on its competitiveness in terms of cost, performance, and acceptance compared to a thermoformed brace. To this purpose, the thermoformed brace of a volunteer patient affected by idiopathic scoliosis was reproduced by 3DP using reverse engineering techniques. Material selection, morphological and mechanical characterization of selected materials, as well as cost analysis of the process were carried out. The $3 \mathrm{D}$ printed brace was finally tested with the volunteer patient, and further deformation and morphological analyses were performed. Performance, acceptability, safety, and satisfaction of the brace were evaluated through a short questionnaire, and thermographic imaging was used to check mechanical effectiveness.

\section{Materials and methods}

\subsection{Preliminary material screening}

A preliminary comparison of the most common commercial filaments available for FDM 3DP was performed using a software database (CES Edupack, Granta Design, UK) and the fabricants' datasheets. The following features were assessed 
on a scale from 1 to 3 (acceptable, good, and excellent): durability, printability, toughness, hydrophobicity, chemical inertness, flexibility, and fatigue resistance. Scoliosis braces are typically used over a t-shirt and not directly in contact with the patient's body; nevertheless, biocompatibility is still of concern. For this reason, availability of medical grade filaments was also reported.

\subsection{Reverse engineering and 3D model preparation}

A virtual replica of the custom-thermoformed brace worn by a patient affected by scoliosis was created by reverse engineering. An infrared triangulation scanner (Structure Sensor, Occipital Inc., USA), adequate for orthopedic applications, connected to a computer via USB and controlled via software (Skanect, Occipital Inc., USA) was used [19]. The initial scan was processed with the general-purpose sculpting CAD software Meshmixer (Autodesk, USA).

\subsection{Printer settings and test samples}

A Delta 4070 Pro (Wasp, Italy) FDM printer was used. This machine has a large cylindrical build volume (400-mm diameter and $670-\mathrm{mm}$ height) that well complies with the dimensions of back braces. It is a delta-style printer, preferred for rounded shapes (e.g., cylinders) with respect to Cartesianstyle printers [20]. The printer was equipped with a nozzle with a diameter of $1.2 \mathrm{~mm}$, which was the largest available for filaments of $1.75 \mathrm{~mm}$, with the goal of reducing the printing time as much as possible.

The settings were preliminary tested by printing hemicylinder samples (Fig. 1a and b), representative of a section of the back brace. The hemicylinders of $150-\mathrm{mm}$ diameter, $50 \mathrm{~mm}$ in height, and $2 \mathrm{~mm}$ of thickness were created with the CAD software Autodesk Inventor and exported as STL file with the preset high level of accuracy, thus obtaining 1190 triangles. The dimensions were decided after measuring the section of a real brace on the reverse engineered 3D model. A short straight part of $30 \mathrm{~mm}$ was extruded on an end of the hemicylinder as it is representative of the regions on the back of the brace, where the curvature decreases and tends to zero. The basic printing parameters for the filament were $240{ }^{\circ} \mathrm{C}$ for extruder temperature, $90{ }^{\circ} \mathrm{C}$ for build plate temperature, and $50 \mathrm{~mm} / \mathrm{s}$ for print speed. To evaluate the effect of increasing layer height on the resulting quality and the mechanical properties, three nominal layer thicknesses were considered: 0.4 , 0.6 , and $0.8 \mathrm{~mm}$. The g-code files were prepared with the slicing software Cura (Ultimaker, the Netherlands).

\subsection{Characterization of 3D printed test samples}

Surface analysis of the 3D printed hemicylindrical samples was performed with a FEI Quanta 200 FEG (Thermo Fisher Scientific, USA) scanning electron microscope (SEM) operating at $20 \mathrm{kV}$. The pieces were coated with gold with a sputter coater (SC500, Emscope, UK) before being observed under the microscope.

Tensile properties were evaluated at room temperature by an Instron machine using a load cell of $1 \mathrm{kN}$ and a crosshead speed of $1 \mathrm{~mm} / \mathrm{min}$. Dumbbell shape specimens were cut from the 3D printed samples and from of the thermoformed polypropylene (PP) and polyethylene (PE) braces according to the ASTM D1708 standard. The study was carried out analyzing five samples for each specimen type. The test was performed considering two fiber orientations: longitudinal (L) and transversal $(\mathrm{T}$ ) to the loading direction (Fig. 2a). The tensile modulus was calculated between 0.05 and $0.20 \%$ strain by a linear regression calculation. Then, mean values and standard deviations were calculated for each specimen series.

Charpy impact tests were performed to compare the energy absorption $\left(E_{c}\right)$ of the different samples, verifying also the effects of fiber orientation and layer thickness. Specimens (13.0-mm wide, 2.5-mm thick, and 60.0-mm long) with a notch depth-to-width ratio of 0.3 were tested at room temperature by using an instrumented apparatus (CEAST Mod. 6545, Instron, Italy) equipped with a Charpy pendulum hammer, setting an impact energy of $3.6 \mathrm{~J}$ and an impact speed of 1 $\mathrm{m} / \mathrm{s}$. Also impact tests were performed considering two
Fig. 1 a Sliced CAD hemicylindrical model ready for printing, where the model is depicted in red and the brim support in blue on the grid of the printing bed, and $\mathbf{b}$ printed PETG sample a)

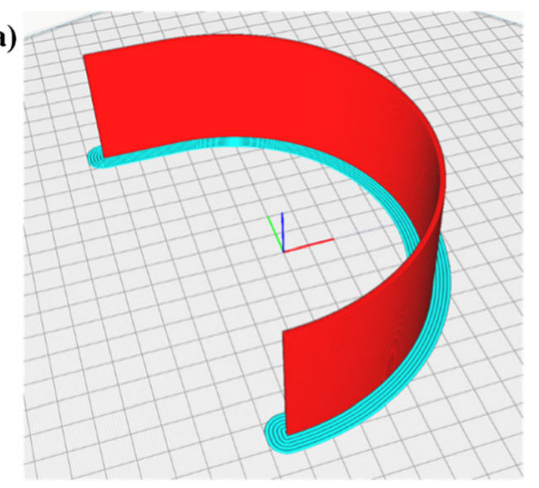

b)

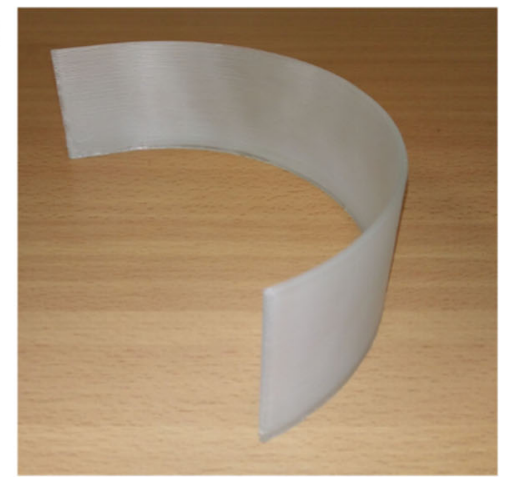


Fig. 2 Fiber orientation with respect to force direction in dumbbell specimens for tensile tests (a) and in V-notched specimens for impact tests (b) a)

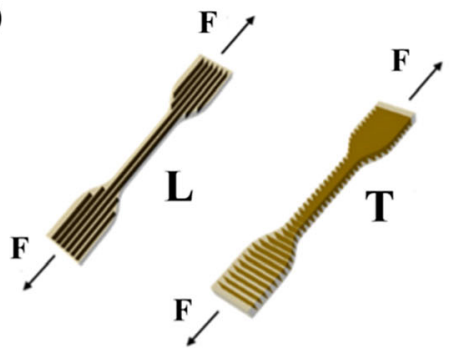

b)

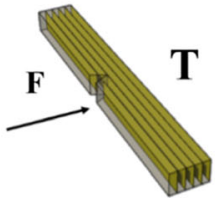

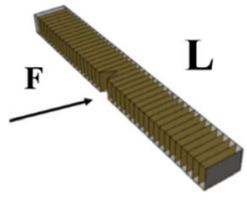

different fiber orientations: longitudinal (L) and transversal (T) to the impact force (Fig. 2b). Each set consisted of five specimens for a given material sample, which enabled to compute average and standard deviations of $E_{c}$.

\subsection{Cost model}

A cost model was developed by combining different cost calculation models described in the literature, adapting it for the specific case of back braces [21, 22]. The model parameters included printer type, materials, and back brace geometry.

The overall cost ( $C_{\text {brace }}[€]$ ) was estimated by accumulation of four subcosts: machine purchase cost $(P)$, machine operation cost $(O)$, material cost $(M)$, and labor cost $(L)$, as shown in Eq. 1.

$C_{\text {brace }}=P+O+M+L$

We considered the total build time $\left(T_{\mathrm{b}}[\mathrm{h}]\right)$ depending on processing time $\left(T_{s}\right)$ and delay time $\left(T_{d}\right)$, where $T_{d}$ is the accumulation of delays before and after printing each layer $\left(L_{p}\right)$ and start-up time $\left(T_{\text {startup }}\right)$.

$T_{b}=T_{d}+T_{s}=\left\{L_{p} \times\left(T_{\text {pre }}+T_{\text {post }}\right)+T_{\text {startup }}\right\}+T_{s}$

$P$ was thus computed as the product of $T_{b}$ and purchase cost $\left(P_{c}[€]\right)$ normalized over useful hours, as shown in Eq. 3. We assumed that orthotists would use the machine $20 \%$ of its useful life ( $Y_{\text {life }}$ [years]), due to their weekly schedule.

$P=\frac{T_{b} \times P_{c}}{0.2 \times 24\left[\frac{\text { hours }}{\text { day }}\right] \times 365\left[\frac{\text { days }}{\text { year }}\right] \times Y_{\text {life }}}$

The machine operation cost $(O)$ was computed as the product of operation rate $\left(C_{o}[€ / \mathrm{h}]\right)$ and build time $\left(T_{b}\right)$, as shown in Eq. 4. $C_{o}$ is an empirically determined constant, correlated to maintenance, consumption of utilities, and cost of space.

$O=T_{b} \times C_{o}$

The material subcost $(M)$ was computed on the material purchase cost $\left(C_{m}[€ / \mathrm{kg}]\right)$. In particular, $C_{m}$ is weighted by the support structure factor $\left(K_{s}\right)$, which captures the percentage of additional material for support structures. $K_{s}$ is equal to 1 without supports, but typically ranges from 1.1 to 1.5 (i.e., 10 to $50 \%$ for supports). The other factors $N, \nu$, and $\rho$ represent number of parts, part volume $\left[\mathrm{cm}^{3}\right]$, and material density $\left[\mathrm{kg} / \mathrm{cm}^{3}\right]$, respectively.

$M=K_{s} \times N \times v \times \rho \times C_{m}$

Finally, labor cost $(L)$ was computed as product of labor time $\left(T_{l}[\mathrm{~h}]\right)$ and labor rate $\left(C_{l}[€ / \mathrm{h}]\right)$, representing the salary of the orthopedic technician (Eq. 6).

$L=T_{l} \times C_{l}$

\subsection{Pilot patient case}

The printed brace was tested by a patient affected by scoliosis, who had been already using the corresponding customthermoformed brace for 1 year. The patient wore the $3 \mathrm{D}$ printed brace continuously for 2 weeks, with a clinical indication to wear the brace for at least $22 \mathrm{~h}$ per day like the traditional one. At the end of the 2 weeks, the patient completed a short questionnaire about acceptance, safety, and satisfaction of the $3 \mathrm{D}$ printed brace, compared with the traditional brace produced by thermoforming. Moreover, thermographic images were obtained using an infrared thermal camera (One, Flir, USA) to objectively compare braces performances [18]. This study was performed in accordance with the Declaration of Helsinki, and the protocol was approved by the IRCCS E. Medea Ethics Committee. The patient gave written informed consent in accordance with the Declaration of Helsinki.

\subsection{Analysis of the back brace after the pilot patient test}

3D scanning of the brace after use was performed with a commercial scanner (Sense ${ }^{\mathrm{TM}}$ 3D scanner, 3D Systems Inc., USA). The 3D scanner was operated in the handheld mode, held at a suitable height and fixed distance $(0.5 \mathrm{~m})$, and moved around the brace. 
The captured 3D image was exported to Rhinoceros CAD Software (Robert McNeel \& Associates, USA) to extract the final 3D model. A Boolean difference operation was applied between the $3 \mathrm{D}$ model of the brace before and after use. The resulting object surface encloses a volume which is the volume belonging to the brace after use but not to that of the original 3D model. For illustrative purposes, the resulting surface was used to create a colored deformation map on the $3 \mathrm{D}$ model of the original brace. Moreover a morphological analysis of the back brace after the pilot patient test has been conducted by SEM. Two samples have been cut from the most deformed zone as indicated in Fig. 10, and they have been analyzed by SEM as described in paragraph 2.4 .

\section{Results}

\subsection{Preliminary material screening}

Common materials used in the thermoforming of braces include polyethylene (PE), polypropylene (PP), and polyethylene foam.

Among the materials listed in Table 1, polylactic acid (PLA) and polyethylene terephthalate glycol-modified (PETG) show the best combination of mechanical properties, processing parameters and low purchase cost; in particular, PETG presents mechanical properties more appropriate for the application (e.g., toughness and hydrophobicity). Therefore, a commercial 1.75-mm diameter PETG filament (Zhuhai Sunlu Industrial Co., China) was selected for the realization of the $3 \mathrm{D}$ printed back brace.

\subsection{Reverse engineering of the traditional scoliosis brace}

The initial 3D scan of the brace took $1 \mathrm{~min}$. The mesh was repaired, and the model was cleaned by removing artifacts. The shape of the frontal straps was removed from the external surface. The inner surface was then used as reference to generate the correct external surface. Finally, the model was completed by deleting the internal surfaces, by cropping the border and by applying a uniform thickness of $2.2 \mathrm{~mm}$ to produce the final solid model. Figure 3 shows the main steps required to obtain the 3D model ready for the slicing software. One hour of working was necessary for the model preparation.

\subsection{Printer settings}

The printer settings in terms of print speed, nozzle temperature, bed temperature, layer height, and extrusion rate were experimentally determined by printing hemi-cylindrical samples (Fig. 1a-b).

Layer height has a direct impact on the printing duration: 23,15 , and $11 \mathrm{~min}$ are required to print hemicylinders with 0.4-, 0.6-, and 0.8-mm layer height, respectively.

\subsection{Characterization of 3D printed test samples}

Figure 4 shows the SEM images of the PETG samples surface with different layer thicknesses and of a thermoformed PP sample. At $\times 100$ magnification (Fig. 4a, c, and e), the PETG samples present a uniform surface finishing, without evident defects (e.g., holes or inclusions) for all the three layer

Table 1 Preliminary comparison and assessment of typical materials available for FDM 3D printers, using data from the database CES EduPack (Granta Design, UK) and filament datasheets.

\begin{tabular}{|c|c|c|c|c|c|c|c|c|}
\hline Material & ABS & PLA & PETG & Nylon & ASA & $\mathrm{PC}$ & PP & PVA \\
\hline Ultimate strength $[\mathrm{MPa}]$ & 40 & 65 & 53 & $40-85$ & 55 & 72 & 32 & 78 \\
\hline Durability & $\checkmark \checkmark \checkmark$ & $\checkmark$ & $\checkmark \checkmark \checkmark$ & $\checkmark \checkmark \checkmark$ & $\checkmark \checkmark \checkmark$ & $\checkmark \checkmark \checkmark$ & $\checkmark \checkmark \checkmark$ & $\checkmark \checkmark$ \\
\hline Coefficient of thermal expansion $\left[\mu \mathrm{m} / \mathrm{m}^{\circ} \mathrm{C}\right]$ & 90 & 68 & 60 & 95 & 98 & 69 & 150 & 85 \\
\hline Price $[€ / \mathrm{kg}]$ & $10-40$ & $10-40$ & $20-60$ & $25-65$ & $38-40$ & $40-75$ & $60-120$ & $40-110$ \\
\hline Printability & $\checkmark \checkmark$ & $\checkmark \checkmark \checkmark$ & $\checkmark \checkmark \checkmark$ & $\checkmark \checkmark$ & $\checkmark \checkmark$ & $\checkmark \checkmark$ & $\checkmark$ & $\checkmark$ \\
\hline Extruder temperature $\left[{ }^{\circ} \mathrm{C}\right]$ & $220-250$ & $190-220$ & $230-250$ & $220-270$ & $235-255$ & $260-310$ & $220-250$ & $185-200$ \\
\hline Bed temperature $\left[{ }^{\circ} \mathrm{C}\right]$ & $95-110$ & $45-60$ & $75-90$ & $70-90$ & $90-110$ & $80-120$ & $85-100$ & $45-60$ \\
\hline Toughness & $\checkmark \checkmark \checkmark$ & $\checkmark$ & $\checkmark \checkmark$ & $\checkmark \checkmark$ & $\checkmark \checkmark \checkmark$ & $\checkmark \checkmark \checkmark$ & $\checkmark \checkmark \checkmark$ & $\checkmark$ \\
\hline Hydrophobicity & $\checkmark$ & $\checkmark$ & $\checkmark \checkmark \checkmark$ & $\checkmark$ & $\checkmark$ & $\checkmark$ & $\checkmark \checkmark \checkmark$ & $\checkmark$ \\
\hline Chemical inertness & $\checkmark$ & $\checkmark$ & $\checkmark \checkmark \checkmark$ & $\checkmark$ & $\checkmark$ & $\checkmark$ & $\checkmark$ & $\checkmark$ \\
\hline Flexibility & $\checkmark$ & $\checkmark$ & $\checkmark \checkmark$ & $\checkmark \checkmark$ & $\checkmark$ & $\checkmark$ & $\checkmark \checkmark \checkmark$ & $\checkmark \checkmark$ \\
\hline Fatigue resistance & $\checkmark$ & $\checkmark$ & $\checkmark \checkmark \checkmark$ & $\checkmark \checkmark \checkmark$ & $\checkmark$ & $\checkmark \checkmark \checkmark$ & $\checkmark \checkmark \checkmark$ & $\checkmark \checkmark \checkmark$ \\
\hline Medical grade & NA & A & A & NA & NA & A & A & A \\
\hline
\end{tabular}

$A B S$ Acrylonitrile butadiene styrene; $P L A$ polylactic acid; $P E T G$ polyethylene terephthalate glycol-modified; $A S A$ acrylonitrile styrene acrylate; $P C$ polycarbonate; $P P$ polypropylene; $P V A$ polyvinyl alcohol; $N A$ not available; $A$ available. Qualitative scale: $\checkmark$ acceptable, $\checkmark \checkmark$ good, $\checkmark \checkmark \checkmark$ excellent 
Fig. 3 Reverse engineering steps on the existing patient-specific thermoformed brace. The initial mesh was repaired and artefacts were removed (a), frontal straps were removed from the external surface (b), and the new outer surface was regenerated using as reference the inner surface, which was then removed (c). Finally, the border was cropped and a uniform thickness of $2.2 \mathrm{~mm}$ was applied (d)
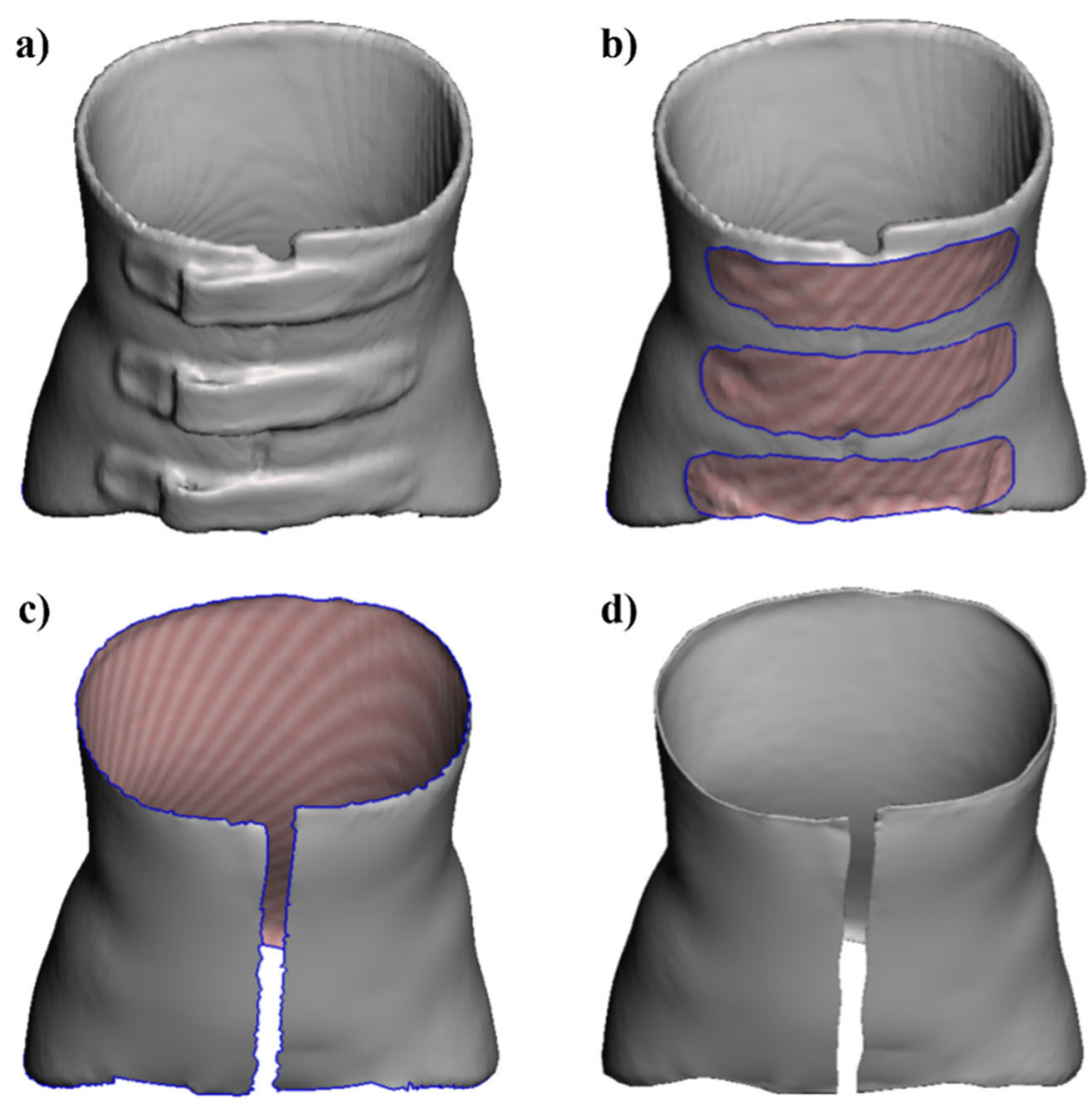

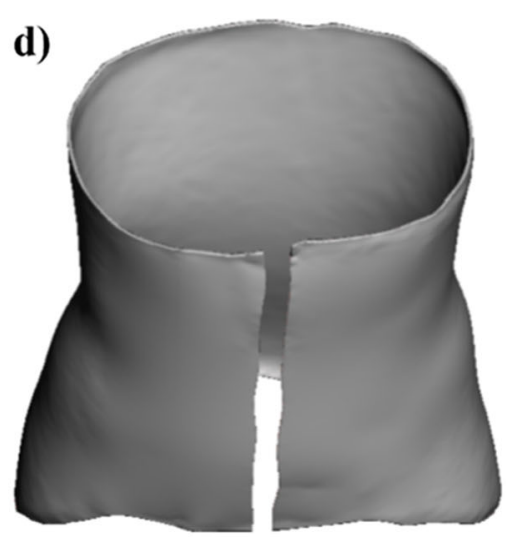

heights. The images at higher resolution $(\times 1000$ - Fig. 4b, d, and $\mathrm{f}$ ) also revealed that all samples presented a correct continuous interface between layers.

An extensive experimental section was designed to study the effects of layer thickness on ultimate strength and elastic modulus of printed specimens at a range of layer thicknesses.
Stress-strain $(\sigma-\varepsilon)$ curves of 3D printed PETG samples are reported in Fig. 5a and b. The mechanical characteristics of 3D printed specimens were compared with samples obtained from thermoformed PE and PP sheets. Given the stress-strain curves, it was possible to calculate some significant parameters, which characterize each sample such as the tensile
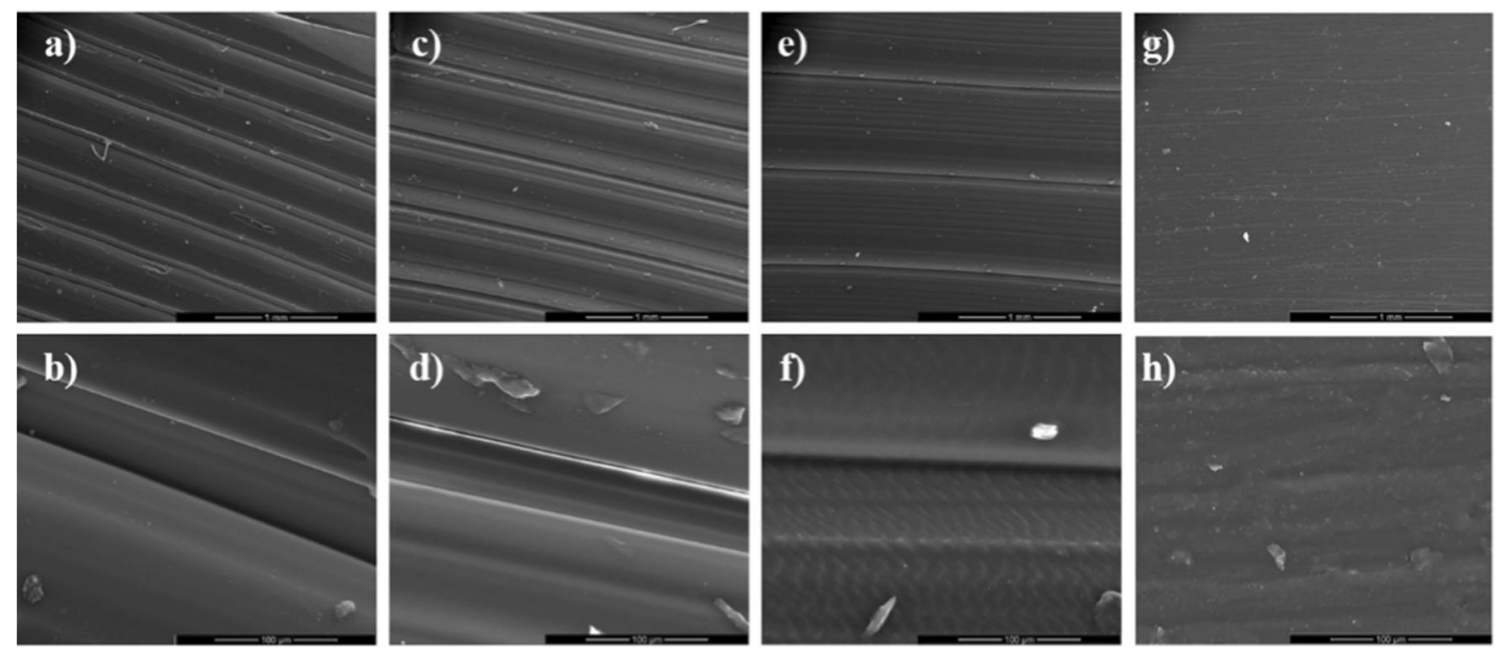

Fig. 4 Scanning electron microscopy image of 3D printed PETG samples and PP thermoformed sample showing roughness and layer interfaces quality at $\times 100(\mathbf{a}, \mathbf{c}, \mathbf{e}, \mathbf{g})$ and at $\times 1000(\mathbf{b}, \mathbf{d}, \mathbf{f}, \mathbf{g})$. PETG samples

with different layer heights (a-b $0.4 \mathrm{~mm} ; \mathbf{c}-\mathbf{d} 0.6 \mathrm{~mm}$; $\mathbf{e}-\mathbf{f} 0.8 \mathrm{~mm}$ ) and reference thermoformed PP $(\mathbf{g}, \mathbf{h})$ 
Fig. 5 a Stress vs strain curves for 3D printed PETG samples tested in $\mathrm{L}$ direction compared with thermoformed samples obtained from a commercial back brace; $\mathbf{b}$ elastic region of the stress-strain curves
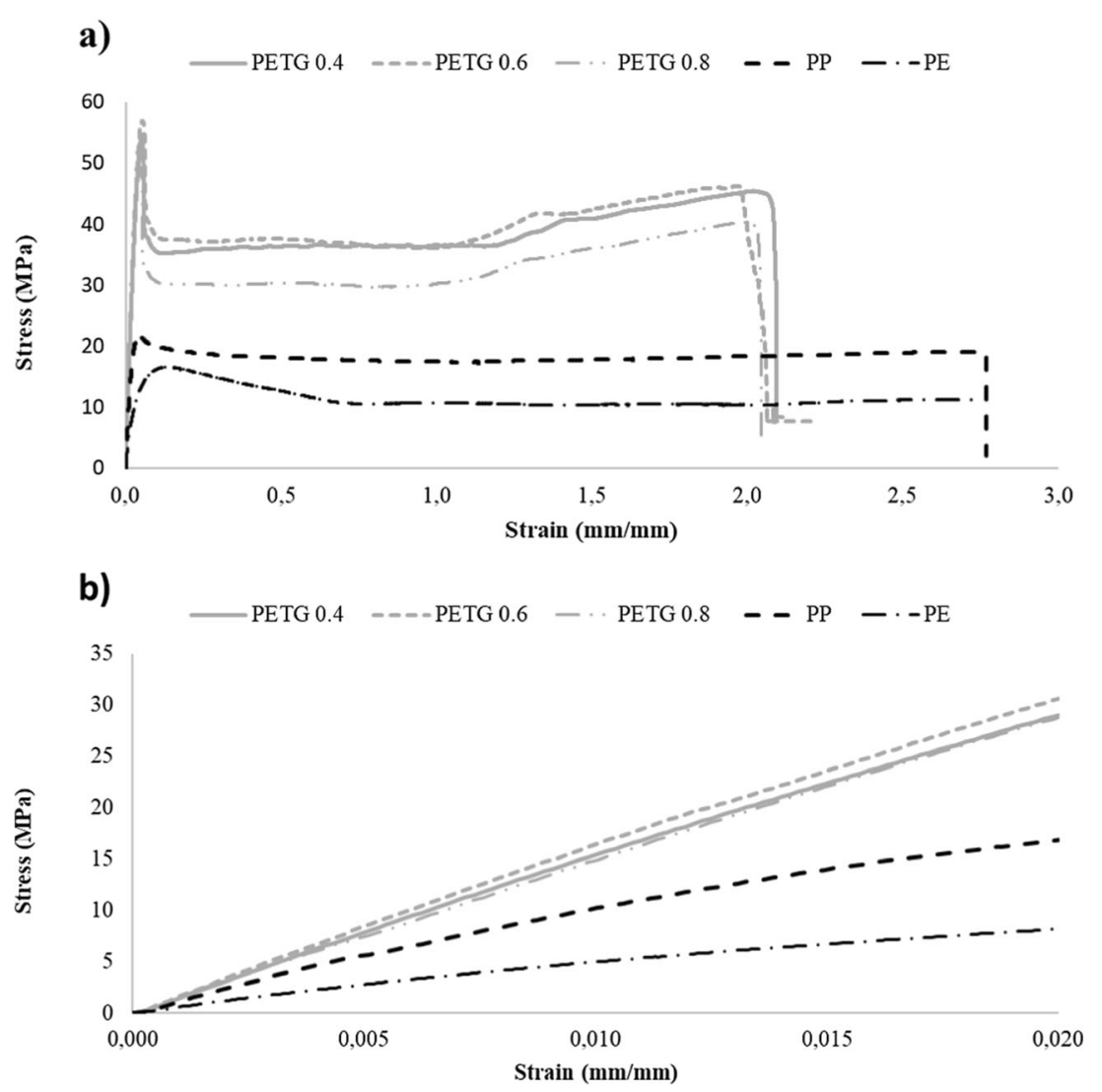

strength $\left(\sigma_{\max }\right)$ elongation at break $\left(\varepsilon_{\max }\right)$ and the Young modulus (E'). From the $\sigma-\varepsilon$ curve depicted in Fig. 5a, it is possible to assess that $3 \mathrm{D}$ printed PETG in L direction showed a typical ductile behavior similar to the one of PP and PE polymers. All the samples exhibit at first a quasi-linear increase of stress as function of strain. After this elastic area, the samples undergo plastic deformation, and stress increases less rapidly until the point of maximum stress $\left(\sigma_{\max }\right)$. Finally, stress decreases as strain increases, until the specimen reaches failure $\left(\varepsilon_{\max }\right)$. Figure $5 \mathrm{~b}$ reports the elastic part of the $\sigma-\varepsilon$ curve to better understand the different mechanical behavior of the analyzed materials. PETG showed higher elastic modulus in respect to the other two polymers and lower deformation at break. This means that the back brace 3D printed in PETG results stiffer if compared with the thermoformed $\mathrm{PP}$ and $\mathrm{PE}$. In particular, $\mathrm{PE}$ showed the lowest elastic modulus, but the highest value of elongation at break that resulted to be one order of magnitude higher if compared with the other materials, which is out of scale in Fig. 5a with a value of about $13 \mathrm{~mm} / \mathrm{mm}$.

To better understand the correlation between different thicknesses and mechanical properties, mean and standard deviation of test results have been reported in Fig. 6. The data show the large variation of properties of samples between $\mathrm{L}$ and $\mathrm{T}$ direction. Regarding the test in $\mathrm{L}$ direction (Fig. 6a, b, and c), the average values among the selected layer heights showed no large discrepancies. The elastic modulus averages varied from $1.48 \pm 0.05 \mathrm{GPa}$ of the $0.6-\mathrm{mm}$ samples to the $1.37 \pm 0.07 \mathrm{GPa}$ of the $0.4-\mathrm{mm}$ ones, while tensile strength varied from $56.26 \pm 0.57 \mathrm{MPa}$ of the $0.6-\mathrm{mm}$ samples to the $48.30 \pm 7.64 \mathrm{MPa}$ of the $0.4-$ $\mathrm{mm}$ ones. Regarding the elongation at break $0.4-\mathrm{mm}$ samples showed the highest value of $\varepsilon_{\max }$ equal to $2.11 \pm 0.108$. Thus, it is possible to assess that PETG samples, tested in $\mathrm{L}$ direction, produced higher values of E' and $\sigma_{\max }$ close to the declared values of the datasheets of the bulk material $\left(\mathrm{E}^{\prime}=2.1 \mathrm{GPa}, \sigma_{\max }=50 \mathrm{MPa}\right)$. On average, the mechanical property values of $3 \mathrm{D}$ printed samples in $\mathrm{L}$ direction resulted comparable with the properties of PP and PE samples. In particular, PETG resulted similar in terms of elastic modulus and elongation at break with PP, but more rigid and brittle if compared with PE. When the force applied was transversal (T) to the fibers (Fig. 6d, e, and f) E' and $\sigma_{\max }$ slightly decreased but remained comparable with the values of PP and PE. On the contrary, $\varepsilon_{\max }$ was lower of about two orders of magnitude in respect to the values in $\mathrm{L}$ direction, varying between $0.037 \pm 0.003 \mathrm{~mm} / \mathrm{mm}$ for $0.4-\mathrm{mm}$ sample to $0.019 \pm 0.002 \mathrm{~mm} / \mathrm{mm}$ for $0.6-\mathrm{mm}$ sample.

The impact test results of printed PETG show that, by increasing the layer thickness, the absorbed energy $\left(\mathrm{E}_{\mathrm{c}}\right) \mathrm{de}-$ creases in both cases of fiber orientation. Moreover, the build orientation significantly influenced the resistance, highlighting a remarkable anisotropy. In the case of impact loading 


\section{Longitudinal fibers (L)}

a)

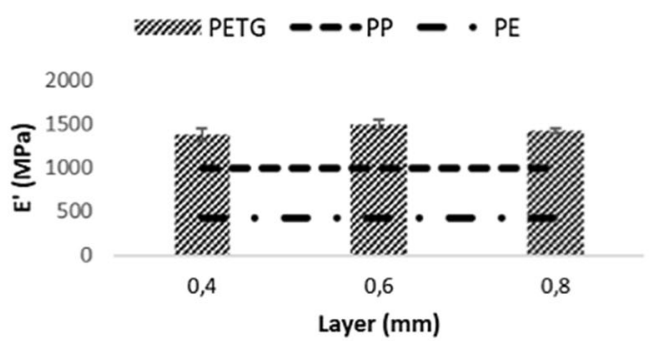

b)

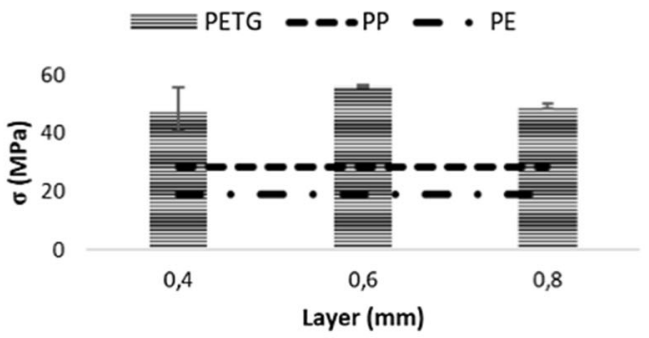

c)

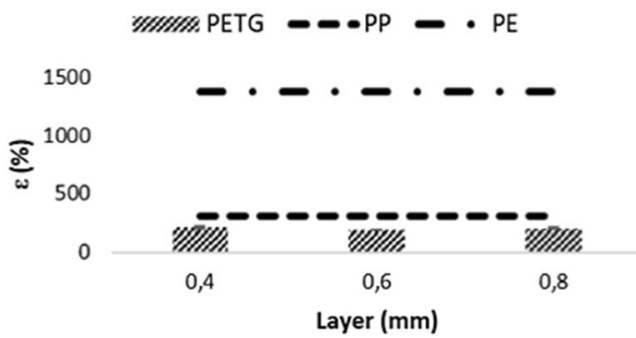

Fig. 6 Results of tensile tests on dumbbell shape specimens. Effect of layer thickness and fiber orientation on printed PETG samples compared with thermoformed PP and PE samples. $\mathbf{a}, \mathbf{b}$, and $\mathbf{c}$ The tests with fibers

parallel to the fibers $(\mathrm{L})$, the absorbed energy $\left(\mathrm{E}_{\mathrm{c}}\right)$ was about 2 orders of magnitude lower than the one measured for the transverse direction (T), as reported in Table 2. Tests have been performed also on PP and PE, but the samples did not

Table 2 Results of absorbed energy $\left(\mathrm{E}_{\mathrm{c}}\right)$ in Charpy impact tests on the $\mathrm{V}$-notched specimens of 3D printed PETG samples with the two fiber orientations (parallel to loading $\mathrm{L}$ and transversal T). The values for PP d)

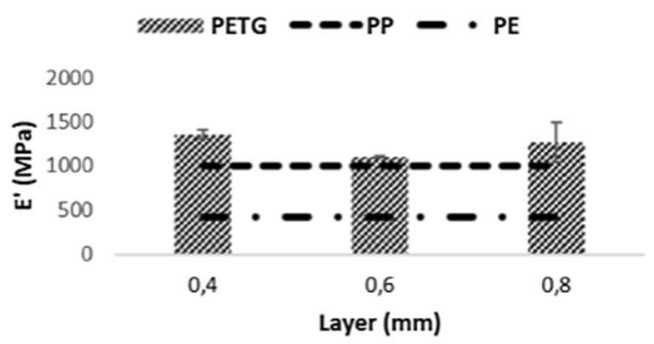

e)

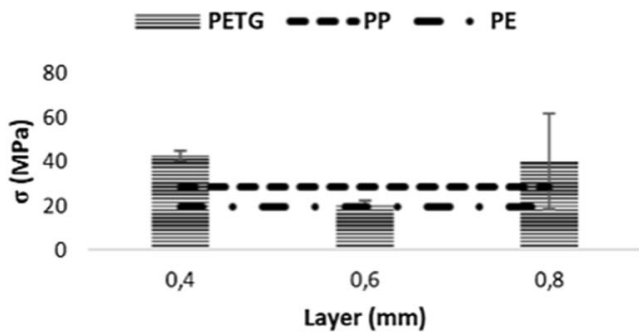

f)

\% PETG

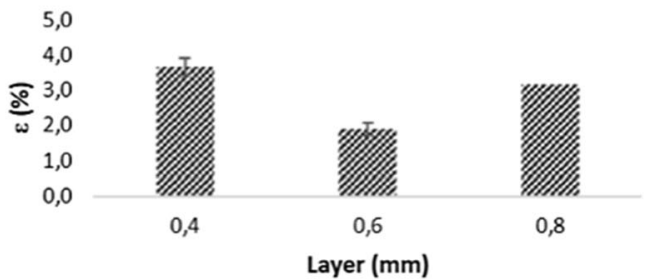

longitudinal to the loading direction (L); $\mathbf{d}, \mathbf{e}$, and $\mathbf{f}$ the tests with fibers in the transverse direction $(\mathrm{T})$

break because the impact energy was below the value necessary for fracture [23]. For this reason, Table 2 reports literature results which are one order of magnitude higher than the best PETG.

and PE were taken from literature ${ }^{19}$, because the specimens did not break in our experiments for limited impact energy

\begin{tabular}{llll}
\hline Material & Fiber orientation & Layer thickness $[\mathrm{mm}]$ & Absorbed impact energy $\left(\mathrm{E}_{\mathrm{c}}\right)\left[\mathrm{J} / \mathrm{m}^{2}\right]$ \\
\hline PETG & $\mathrm{L}$ & 0.4 & $3.16 \pm 0.45$ \\
& & 0.6 & $2.88 \pm 0.44$ \\
& $\mathrm{~T}$ & 0.8 & $1.61 \pm 0.37$ \\
& & 0.4 & $122.27 \pm 1.60$ \\
& & 0.6 & $112.04 \pm 4.33$ \\
$\mathrm{PP}$ & - & 0.8 & $86.91 \pm 6.05$ \\
$\mathrm{PE}$ & - & - & 6000 \\
\hline
\end{tabular}




\subsection{Cost model}

Table 3 shows a detailed breakdown of the costs and the printing parameters associated with the 3DP process when printing the back brace on the WASP Delta 4070 PRO FDM printer. The machine purchase price $(\mathrm{Pc})$ was $7000 €$ and its useful life (Ylife) is expected to be 7 years. Tb was obtained by Eq. 2, where $T_{\text {pre }}$ and $T_{\text {post }}$ are equal to zero for FDM technology, as the printing is continuous, while $\mathrm{T}_{\text {startup }}$ is about 30 minutes for calibration and warm-up. In this study, we set the scanning time $\left(T_{s}\right)$ equal to the estimated time needed to print the back brace, obtained from the g-code file, as it strongly depends on process parameters (e.g., layer height and printing speed).

To compute the machine operative cost $\mathrm{O}$, we empirically determined an operation rate $\left(\mathrm{C}_{\mathrm{o}}\right)$ of $4.75 € / \mathrm{h}$. The material $\operatorname{cost}(\mathrm{M})$ was computed with a support structure factor equal to 1.2 and we expect to print one brace at a time $(N=1)$. Part volume was obtained from the CAD file $(417 \mathrm{~cm} 3)$. The PETG filament had a cost of $30 € / \mathrm{kg}$, while its declared density was $1.27 \mathrm{~g} / \mathrm{cm}^{3}$. Finally, the labor rate for the orthopedic technician is $25 € / \mathrm{h}$ and labor time was $2 \mathrm{~h}$, including model preparation, printer setup and manual operations on the printed back brace.

By combining the different contributes, the overall cost (C) of the back brace would be $109.57,94.93$, and $90.05 €$ when

Table 3 Detailed cost breakdown of the proposed cost model for producing a back brace in PETG with an FDM 3D printer, in the three selected cases of $0.4,0.6$, and $0.8 \mathrm{~mm}$ for the layer height

\begin{tabular}{llll}
\hline & Value & \\
\cline { 2 - 4 } & 0.4 & 0.6 & 0.8 \\
\hline Layer height $[\mathrm{mm}]$ & 1.08 & 0.69 & 0.56 \\
\hline Machine cost $-\mathrm{P}[€]$ & 8.30 & 5.30 & 4.30 \\
Build time $-\mathrm{T}_{\mathrm{b}}[\mathrm{h}]$ & 7000 & 7000 & 7000 \\
Purchase price $-\mathrm{P}_{\mathrm{c}}[€]$ & 7 & 7 & 7 \\
Expected life $-\mathrm{Y}_{\text {life }}[$ years $]$ & 39.43 & 25.18 & 20.43 \\
Operative cost $-\mathrm{O}[€]$ & 4.75 & 4.75 & 4.75 \\
Operation rate $-\mathrm{C}_{\mathrm{o}}[€ / \mathrm{h}]$ & 8.30 & 5.30 & 4.30 \\
Build time $-\mathrm{T}_{\mathrm{b}}[\mathrm{h}]$ & 19.07 & 19.07 & 19.07 \\
Material cost $-\mathrm{M}[€]$ & 1.2 & 1.2 & 1.2 \\
Support material factor $-\mathrm{K}_{\mathrm{s}}$ & 1 & 1 & 1 \\
Number of parts $-\mathrm{N}$ & 417 & 417 & 417 \\
Part volume $-\nu\left[\mathrm{cm}^{3}\right]$ & 30 & 30 & 30 \\
Material rate per unit weight $-\mathrm{C}_{\mathrm{m}}[€ / \mathrm{kg}]$ & 1.27 & 1.27 & 1.27 \\
Material density $-\rho\left[\mathrm{g} / \mathrm{cm}^{3}\right]$ & 50.00 & 50.00 & 50.00 \\
Labor cost $-\mathrm{L}[€]$ & 2 & 2 & 2 \\
Labor time $-\mathrm{T}_{1}[\mathrm{~h}]$ & 25 & 25 & 25 \\
Labor rate $-\mathrm{C}_{1}[€ / \mathrm{h}]$ & 109.57 & 94.93 & 90.05 \\
Overall Cost $-\mathrm{C}[€]$ & & & \\
\hline & & &
\end{tabular}

printed with a layer height of $0.4 \mathrm{~mm}, 0.6 \mathrm{~mm}$, and $0.8 \mathrm{~mm}$, respectively.

\subsection{Back brace manufacturing and patient test}

The final back brace was printed in PETG with a layer height of $0.6 \mathrm{~mm}$, to comply with the time limit of a working day, having a printing time of $4 \mathrm{~h}$ and $33 \mathrm{~min}$. The brace was printed with two walls equal to the nozzle diameter and printed upside-down to limit the required supports (Fig. 7ac). A small support was produced with a single line deposition with "zig-zag" path, as a compromise between good stability and ease to remove the supports from the brace. The bounding box containing the brace measures $302 \mathrm{~mm}$ in vertical direction, $298 \mathrm{~mm}$ in the lateral direction and $216 \mathrm{~mm}$ in the frontto-back direction.

The brace was then removed from the 3D printer build plate, and the supports were easily detached manually. The mass of the final brace is $530 \mathrm{~g}$ after removing the supports (16 g), comparable with the $510 \mathrm{~g}$ of the thermoformed version, both considered without the closure straps. An experienced orthotist completed the finishing of the borders and

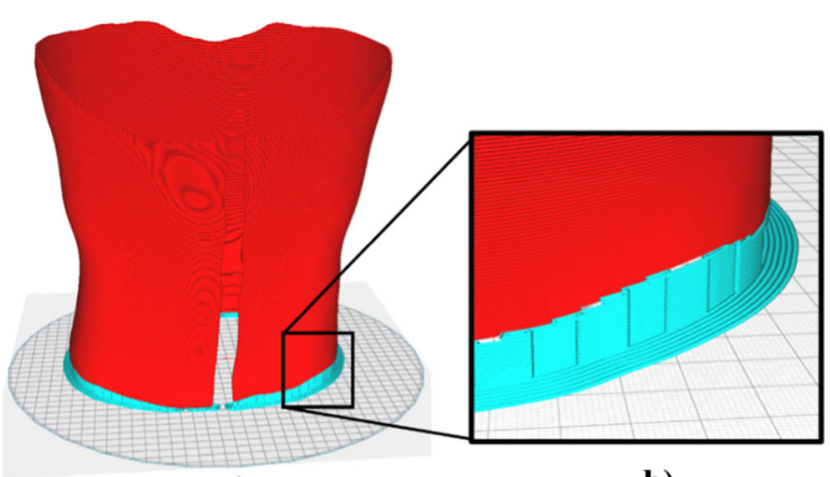

a)

b)

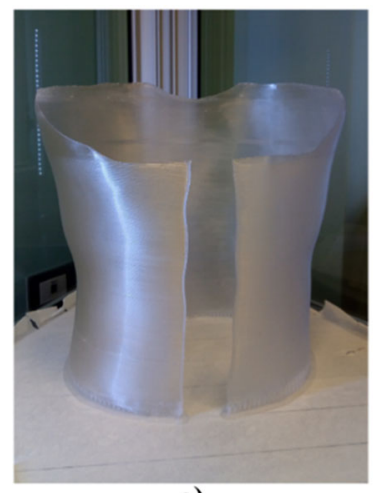

c)

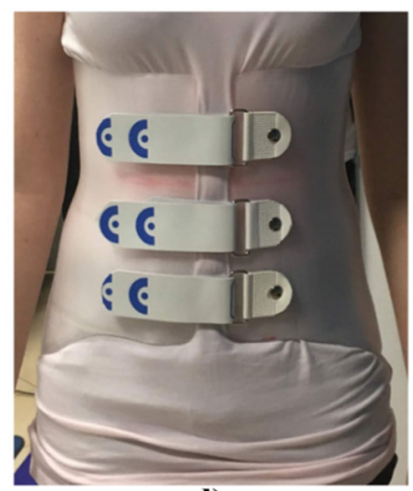

d)
Fig. 7 Brace 3D printing and patient test. Printing setup of the brace in Ultimaker CURA (a), where the model is depicted in red and the supports in blue. A detailed view of the "zig-zag" path for the support structure (b). 3D printed brace on build plate inside the large 4070 delta printer (c) and worn by the volunteer patient after final refinement by the orthotist (d) 
Fig. 8 Infrared thermographic images of the posterior side of the patient wearing the traditional thermoformed brace (a) and the 3D printed brace (b) with relative temperature scale bar

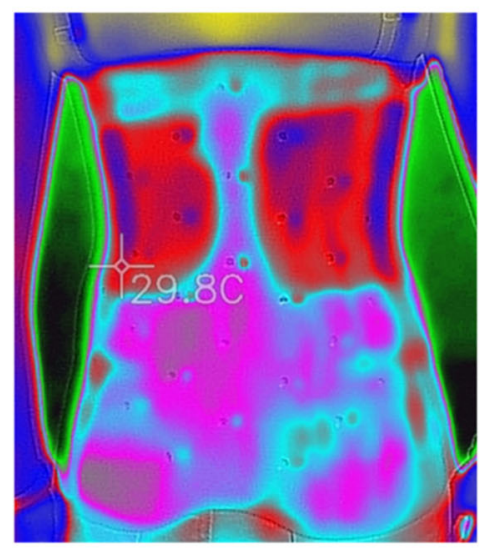

a)

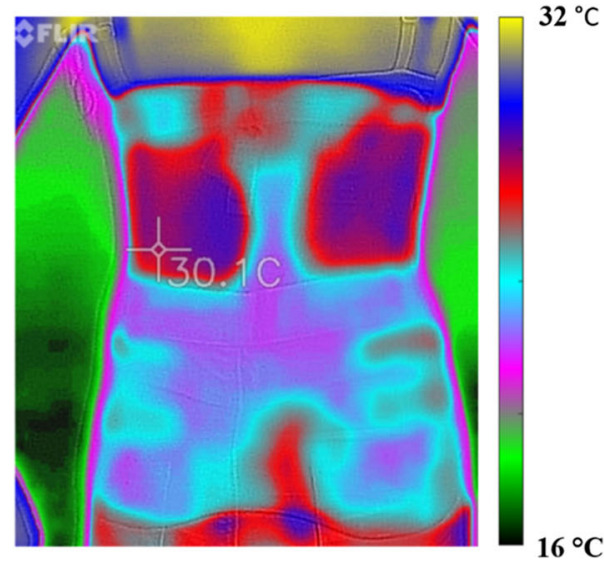

b) applied the closure straps, using routine tools (grinder, drill, and riveter) available in orthopedic workshops, for additional 30 min. Finally, the brace was worn by the subject, and minor adjustments were done.

The patient was compliant and wore the 3D printed brace continuously for 2 weeks. With respect to the traditional thermoformed orthosis, the patient reported less discomfort and an increased supporting action due to the higher stiffness of the brace. An improved wearability and a greater ease in performing activities of daily living were also conveyed through the short questionnaire. In contrast, the patient reported the feeling that the 3D printed brace was more brittle compared with the traditionally manufactured orthosis. Infrared thermography images did not show significant differences in superficial temperature between the two brace types (Fig. 8a and b).

A deformation analysis of the 3D printed back brace after the patient test has been conducted in order to understand any macrorelaxation/deformation of the structure (Fig. 10). The back brace after the patient test have been scanned and compared with the 3D back brace before the test.

In Fig. 9, the different colors indicate the distribution of deformations over the entire structure after use. It is quite evident that the deformations are homogeneous and symmetrical on the whole brace. In particular, all the contours are deformed outward due to the tensions produced during use (green area). High levels of deformation are located at the rear where the brace is most mechanically stressed during the wear process. Localized deformations are also present on the front of the brace. The stress produced by the presence of the closing straps is probably responsible for these deformations. In any case, the total deformations are small and unable to compromise the structural functionality of the brace. It can be concluded that the $3 \mathrm{D}$ printed structure is able to properly resist the fatigue produced during use by the patient, which is a very important result. In fact, an uncontrolled mechanical relaxation of the structure would have compromised the functionality of the orthopedic device.

Starting from these results, an analysis of the morphology of the brace surface was conducted. The aim was to verify the state of the structure at the microscopic level near the highest deformations. Figure 10 shows SEM images of material samples taken from two different positions as showed from in Fig. 10, corresponding to two extreme deformations (outward and inward).
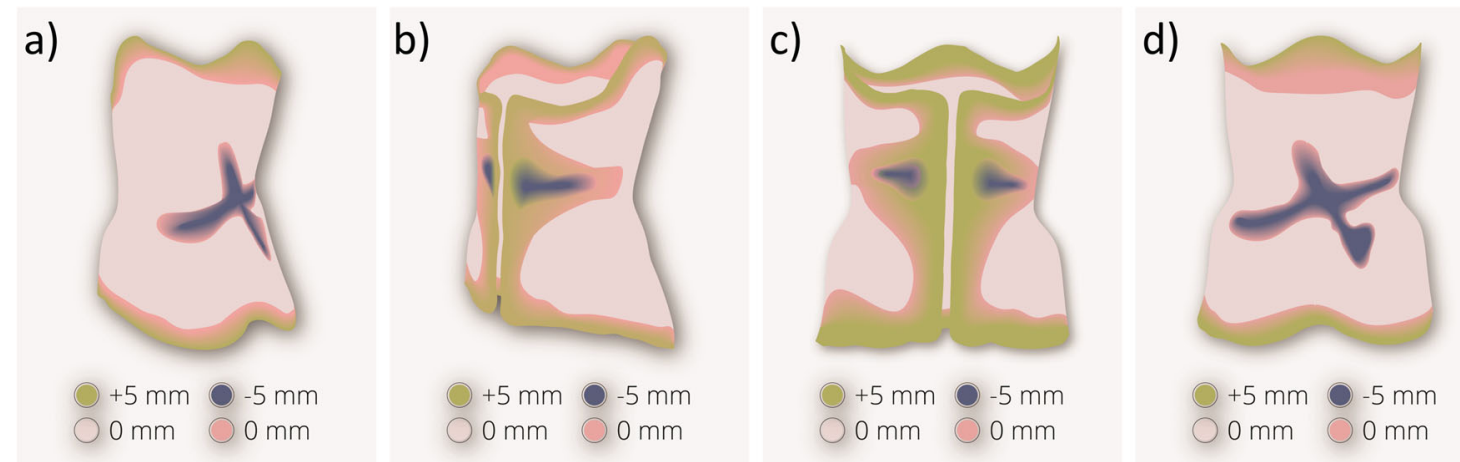

Fig. 9 Deformation analysis of the 3D printed back brace after the patient test $\mathbf{a}$ back lateral view; $\mathbf{b}$ front lateral view, $\mathbf{c}$ front view, and $\mathbf{d}$ back view of the back brace. Positive deformation with outward direction (green), null deformation (pink), and negative deformation (blue) 
Fig. 10 SEM images of samples cut from the $3 \mathrm{D}$ printed back brace after the patient test in the two extreme deformation areas, at $\times 100$ of magnification (Scale bar represents $1 \mathrm{~mm}$ )

From the SEM images shown in Fig. 10, it is evident that after two weeks of testing the structure of the brace at the microscopic level is intact. In fact, there are no visible microcracks or delamination between the different printed layers. This means that the structure of the brace did not change during use, maintaining its mechanical integrity.

Even comparing the images of Fig. 10 with those of Fig. 4, there are no differences. The only difference is given by the presence of small fragments or dirt particles present on the surface of the brace. These are probably the result of daily patient use.

\section{Discussion}

\subsection{Reverse engineering and 3D printing}

The 3D model preprocessing phases (smoothing, artifact removal, external surface creation, and thickening) of the brace took approximately $1 \mathrm{~h}$, which is comparable with the time required to prepare a CAD model and set the CAM module for the milling machine, obtaining the positive mold in the current production process.

Printing tests showed that layer height greatly affected results in terms of printing duration, while surface quality and mechanical parameters were only partially influenced by this parameter. However, this is a crucial aspect to consider when scaling up the production and further tests are required to establish the optimal tradeoff between printing duration and product quality.

The overall production time from initial scanning to delivery to the patient took approximately a full working day, similarly to what is required by the modern thermoforming process. However, the total man-hours are reduced because of the minimal supervision necessary during the $3 \mathrm{D}$ printing.

The machinability of the $3 \mathrm{D}$ printed brace was slightly lower than that of traditional thermoformed braces: the jigsaw was not used for the possible delamination of printed layers, and holes for the rivets that hold the closure straps were performed more carefully and with a lower drilling speed. However, no limitations in using the grinder were found, both with sandpaper wheels for removing material and with a felt grinder wheel for smoothing the borders. 


\subsection{Characterization of 3D printed test samples}

The SEM analysis revealed that the 3D printed samples showed a roughness due to the layer-by-layer nature of the $3 \mathrm{D}$ printing technique. However, the surface of the $3 \mathrm{D}$ printed samples resulted acceptable for absence of holes and limited inclusion, if compared with the PP sample. Moreover, the morphological analysis showed that the interface and the surface quality were not dependent from the layer thickness.

As expected, tensile tests results showed that FDM specimens presented different mechanical responses depending on fiber direction (almost not depending on layer height). When the load was applied longitudinally to the fibers, the behavior was comparable to that of PP and PE, thanks to the crosssectional resistance of the fibers. When the fibers were orthogonal to the loading direction, the sample strength depended mainly on the inter-fiber bonding, reducing the overall resistance. However, the higher elastic modulus could be exploited to reduce the brace thickness, improving the overall comfort.

The absorbed energy $(\mathrm{Ec})$ for PETG printed samples resulted to be inversely proportional to layer height and lower than PP and PE. When higher layers are printed for a given dimension, the same layers create a larger macroroughness with possible notch effect on the surface, influencing fracture mechanics. Moreover, Ec for PETG with transverse fibers resulted about two orders of magnitude higher than in longitudinal direction. This confirms the results obtained from the tensile test, providing evidence for the quality of interface bonding between adjacent layers. When the fiber connection has to withstand the load, the adhesion resistance decreases the bulk material properties to the point that the printed components result brittle. Considering that brace replacement must occur both in case of critical breaks with undesired impacts and plastic deformations, PETG is adequate for this application.

\subsection{Cost model}

The cost analysis showed that about half of the cost was attributed to labor cost. This preliminary analysis evidenced also the significant influence of the build time $\left(\mathrm{T}_{\mathrm{b}}\right)$ on the operative costs, highlighting the importance of high printing speeds. Moreover, it has to be noticed that the accurate estimation of operative rate $\left(\mathrm{C}_{\mathrm{o}}\right)$ highly depends on the cost structure of the company that owns the FDM machine. However, the back brace cost resulting from the model is very competitive compared to the production cost of a thermoformed back brace, that typically ranges from 250 to $500 €$ due to the long labor time.

\subsection{Pilot patient case}

The brace was worn successfully for 2 weeks, and the device did not show any sign of delamination. According to the patient, the $3 \mathrm{D}$ printed brace was stiffer than the thermoformed one, with an improved supporting action. The patient also reported an increased sensation of brittleness which caused some slight concern during activities of daily living, with the fear of breaking the brace. However, this did not result in any adverse event during the 2 weeks of test. Longer tests should be performed in future works.

In our study, superficial temperature was used as an indirect indication of brace adhesion and contact, highlighting no significant differences between the 3D printed and the thermoformed device, with negligible temperature differences. Overall, the study showed that the production of 3D printed braces is feasible and was positively assessed by patient and orthotist. Our results provide encouraging preliminary findings and a clear direction for further improving and using virtual modeling and 3D printing in the field of orthotic bracing for the treatment of spine diseases.

The orthopedic physician agreed on the sensation of the patient about the material properties, the brace is effectively stiffer. However, the important feedback regards the good function of a stiffer material for applying a stronger correction, also in those cases of older patients with AIS where the spine becomes less flexible. Moreover, the physician noticed how this solution could be comparable to the stiff braces with metal rods (e.g., Milwaukee and Sforzesco braces) but with the advantage of a reduced mass and an increased acceptability.

The orthopedic technicians who postprocessed the brace found some differences also in terms of machinability. The use of the jig saw had to be avoided, because of possible delamination of the printed layers. The creation of the holes for the rivets that hold the closure straps had to be performed more carefully and with a lower drilling speed. Nevertheless, they found no limitation in using the grinder, both with rough and fine sandpaper wheels for removing material and with a felt grinder wheel for smoothing the border.

Moreover the morphological and deformation analysis of the back brace after the patient test confirm that the brace presented no morphological and structural modification after it use. Small and evenly distributed deformations found on the brace structure ensure its mechanical stability during use.

\section{Conclusions}

In this study, an innovative production process of orthopedic scoliosis braces based on FDM 3D printing is proposed and analyzed. The aim of the manuscript is not limited to the demonstration of the feasibility of the $3 \mathrm{D}$ printing process to produce scoliosis back braces, but it is meant to evaluate the effectiveness and the efficiency of such technology in terms of material behavior, costs, clinical applicability, and patient's satisfaction. 
After a screening of commercial materials, a PETG filament was chosen. Printed samples were characterized in terms of mechanical and morphological properties. PETG samples revealed mechanical properties similar to PP samples in terms of elastic modulus and ultimate tensile strength. The PE samples performed better in terms of elongation at break but resulted less stiff than PETG. A 3D printed brace was produced by reverse engineering based on an existing thermoformed brace. The main differences with respect to the thermoformed brace were related to lower machinability during postprocessing (requiring to be more careful in using the common tools), higher stiffness and higher apparent brittleness. The test on a patient with scoliosis showed both the feasibility of the manufacturing process and the competitiveness in terms of costs using $3 \mathrm{D}$ printing with respect to classic thermoforming procedure.

All the involved people (patient, physician, and technicians) noticed the stiffer behavior of the brace, but they did not evaluate this as a disadvantage. PETG has indeed an elastoplastic behavior with a reduced elongation to rupture in respect to the thermoformed PE. Nevertheless, the brace should not work beyond the yield stress otherwise the shape would be modified and the physician stated that PETG is a good compromise between completely rigid braces with metal rods and softer braces made in PE or PP.

Future studies will need to provide further evidence for long-term reliability of the technology, patient acceptance and clinical efficacy.

Acknowledgments This study was partially supported by a grant of Fondazione Cariplo and Regione Lombardia, through the project EMPATIA@Lecco, by the Italian Ministry of Health (Ricerca Corrente 2020 to Dr. E. Biffi) and by the Italian Ministry of Public Education, University and Research for the PhD scholarship of Dr. Redaelli. The authors would like to thank the orthopedic technicians (from Biomedica su Misura, Brescia, Italy and Ortopedia LABSAN, Lecco, Italy) and the volunteer patient for taking part into the experimentation.

Funding Open access funding provided by Politecnico di Milano within the CRUI-CARE Agreement. This study was partially supported by a grant of Fondazione Cariplo and Regione Lombardia, through the project EMPATIA@Lecco, by the Italian Ministry of Health (Ricerca Corrente 2019 to Eng. G. Reni and Ricerca Corrente 2020 to Dr. E. Biffi) and by the Italian Ministry of Public Education, University and Research for the $\mathrm{PhD}$ scholarship of Dr. Redaelli.

Data availability Data are available on request by sending an email to the corresponding author.

\section{Compliance with ethical standards}

All materials and methods regarding this study have been analyzed and approved by the Ethics Committee of Scientific Institute, IRCCS E. Medea, and all procedures are in agreement with the principles expressed in the Declaration of Helsinki. Participant and parents of the minor patient are informed about the aims and procedures of the study and then are asked to sign an informed consent before starting the baseline evaluation.
Conflict of interest The authors declare that they have no conflict of interest.

Code availability Not applicable

Open Access This article is licensed under a Creative Commons Attribution 4.0 International License, which permits use, sharing, adaptation, distribution and reproduction in any medium or format, as long as you give appropriate credit to the original author(s) and the source, provide a link to the Creative Commons licence, and indicate if changes were made. The images or other third party material in this article are included in the article's Creative Commons licence, unless indicated otherwise in a credit line to the material. If material is not included in the article's Creative Commons licence and your intended use is not permitted by statutory regulation or exceeds the permitted use, you will need to obtain permission directly from the copyright holder. To view a copy of this licence, visit http://creativecommons.org/licenses/by/4.0/.

\section{References}

1. Kuroki H (2018) Brace treatment for adolescent idiopathic scoliosis. J Clin Med 7. https://doi.org/10.3390/jcm7060136

2. Schott C, Zirke S, Schmelzle JM, Kaiser C, I Fernández LA (2018) Effectiveness of lumbar orthoses in low back pain: Review of the literature and our results. Orthop Rev (Pavia) 10. https://doi.org/10. 4081/or.2018.7791

3. Rauch F, Glorieux FH (2004) Osteogenesis imperfecta. Lancet, In

4. Redaelli DF, Biffi E, Colombo G, et al (2018) Current and future manufacturing of chest orthoses, considering the case of osteogenesis imperfecta. In: ASME 2018 International Design Engineering Technical Conferences and Computers and Information in Engineering Conference. pp 1-7

5. Courvoisier A, Drevelle X, Vialle R, Dubousset J, Skalli W (2013) 3D analysis of brace treatment in idiopathic scoliosis. Eur Spine J 22:2449-2455. https://doi.org/10.1007/s00586-013-2881-7

6. Law D, Cheung MC, Yip J, Yick KL, Wong C (2017) Scoliosis brace design: influence of visual aesthetics on user acceptance and compliance. Ergonomics. 60:876-886. https://doi.org/10.1080/ 00140139.2016.1227093

7. Redaelli DF, Storm FA, Biffi E et al (2019) Virtual design process to produce scoliosis braces by additive manufacturing. In: International Conference on Design, Simulation, Manufacturing: The Innovation Exchange. Springer, Berlin, pp 860-870

8. Saleh JM, Dalgarno KW (2010) Cost and benefit analysis of fused deposition modelling (FDM) technique and selective laser sintering (SLS) for fabrication of customised foot orthoses. Innovative Developments in Design and Manufacturing - Advanced Research in Virtual and Rapid Prototyping, In

9. Molnár I, Morovič L (2018) Design and manufacture of orthopedic corset using 3D digitization and additive manufacturing. In: IOP Conference Series: Materials Science and Engineering

10. Jiang J (2020) A novel fabrication strategy for additive manufacturing processes. J Clean Prod 122916

11. Jiang J, Ma Y (2020) Path planning strategies to optimize accuracy, quality, build time and material use in additive manufacturing: a review. Micromachines 11:633

12. Jiang J, Xu X, Stringer J (2019) Optimization of process planning for reducing material waste in extrusion based additive manufacturing. Robot Comput Integr Manuf 59:317-325

13. Banks J (2013) Adding value in additive manufacturing: Researchers in the United Kingdom and Europe look to 3D printing 
for customization. IEEE Pulse 4:22-26. https://doi.org/10.1109/ MPUL.2013.2279617

14. Mertz L (2013) Dream it, design it, print it in 3-D: What can 3-D printing do for you? IEEE Pulse 4:15-21. https://doi.org/10.1109/ MPUL.2013.2279616

15. Li J, Tanaka H (2018) Feasibility study applying a parametric model as the design generator for 3D-printed orthosis for fracture immobilization. 3D Print Med 4. https://doi.org/10.1186/s41205-0170024-1

16. Li J, Tanaka H (2018) Rapid customization system for 3D-printed splint using programmable modeling technique - a practical approach. 3D Print Med 4.

17. Lanzotti A, Martorelli M, Maietta S, Gerbino S, Penta F, Gloria A (2019) A comparison between mechanical properties of specimens 3D printed with virgin and recycled PLA. Procedia CIRP 79:143146. https://doi.org/10.1016/J.PROCIR.2019.02.030

18. Patterson AE, Pereira TR, Allison JT, Messimer SL (2019) IZOD impact properties of full-density fused deposition modeling polymer materials with respect to raster angle and print orientation. Proc Inst Mech Eng Part C J Mech Eng Sci:095440621984038. https:// doi.org/10.1177/0954406219840385
19. Redaelli DF, Gonizzi Barsanti S, Fraschini P, et al (2018) Low-cost $3 \mathrm{D}$ devices and laser scanners comparison for the application in orthopedic centres. ISPRS - Int Arch Photogramm Remote Sens Spat Inf Sci XLII-2:953-960. https://doi.org/10.5194/isprsarchives-XLII-2-953-2018

20. Bell C (2015) 3D Printing with Delta Printers. APRESS, New York

21. Yim S, Rosen D (2012) Build time and cost models for Additive Manufacturing process selection. In: Proceedings of the ASME Design Engineering Technical Conference

22. Ruffo M, Hague R (2007) Cost estimation for rapid manufacturing Simultaneous production of mixed components using laser sintering. Proc Inst Mech Eng Part B J Eng Manuf 221:15851591. https://doi.org/10.1243/09544054JEM894

23. Van Krevelen DW, Nijenhuis K Te (2009) Properties of polymers: their correlation with chemical structure; their numerical estimation and prediction from additive group contributions

Publisher's note Springer Nature remains neutral with regard to jurisdictional claims in published maps and institutional affiliations. 